\title{
Mini-symposium
}

\section{Endothelial function: 20 years of clinical investigation}

l: 1980 Furchgott and Zawadzki described the phenomenon of endothelium dependent relaxation ${ }^{1}$ and identified a novel labile relaxant factor later identified as nitric oxide. A few years earlier John Vane and colleagues $^{2}$ had identified prostacyclin as a potent antiplatelet agent released from endothelial cells. Together these findings stimulated a huge increase in clinical research in vascular mechanisms and endothelial function. Twenty years on it is time to take stock.

The three articles in this mini-symposium place the research on endothelial function in a clinical context. Deanfield and Halcox outline the evidence that changes in endothelium dependent relaxation can be detected even in children and that this may represent the very earliest changes that ultimately lead to atherosclerosis. Hingorani takes this discussion into the realms of nature versus nurture debate by identifying the potential impact of genetic variations in determining key aspects of endothelial function. Finally, Vita addresses the question of whether measures of endothelium dependent relaxation are predictive of cardiovascular events, how changes in more accessible vascular beds such as the forearm relate to changes in the heart, and whether there is a realistic possibility that measures of endothelial function will have a place in the clinic.

Three other areas are emerging that are not covered in detail but warrant consideration. The first is the question of whether circulating endothelial progenitor cells are important in repair processes and to what extent they determine vascular health. The second is the variable use of the term "endothelial function" that is used and understood differently by different individuals; it is increasingly clear that there are so many different functions of the endothelium that the catch-all term may no longer be appropriate. The third relates to the recent observations that endothelium dependent relaxation varies widely within individuals from day to day dependent on other factors such as the state of systemic inflammation ${ }^{3}$ or even in response to mental stress. ${ }^{4}$ It seems that as endothelial biology is becoming very much part of mainstream cardiology there is still more that we do not know than there is that we do know.

Correspondence to: Professor Patrick Vallance, BHF Laboratories, Division of Medicine, University College London London WIP 9LN, UK patrick.vallance@ucl.ac.uk

\section{REFERENCES}

1 Furchgott RF, Zawadzki JV. The obligatory role of endothelial cells in the relaxation of arterial smooth muscle by acetylcholine. Nature 1980;288:373-6.

2 Moncada S, Gryglewski R, Bunting S, et al. An enzyme isolated from arteries transforms prostaglandin endoperoxides to an unstable substance that inhibits platelet aggregation. Nature 1976:263:663-5.

3 Kharbanda RK, Walton B, Allen $M$, et al. Prevention of inflammation-induced endothelial dysfunction: a novel vasculo-protective action of aspirin. Circulation 2002;105:2600-4.

4 Ghiadoni L, Donald AE, Cropley M, et al. Mental stress induces transient endothelial dysfunction in humans. Circulation 2000;102:2473-8.

\section{IMAGES IN CARDIOLOGY}

\section{Right atrial mass: primary angiosarcoma}

A 41 year old man presented with history of shortness of breath and swelling of feet, and was diagnosed with angiosarcoma of the right atrium by biopsy. Subsequently he had complications including pericardial tamponade for which he was treated with a pericardial window and pleural effusions treated with thoracocentesis and pleurodesis. He was referred to our institution for further treatment. The patient received chemotherapy with epirubicin and imatinib mesylate initially, and was subsequently treated with gemcitabine and docetaxel.

A transoesophageal echocardiogram (panel A) was performed and showed extensive involvement of the right
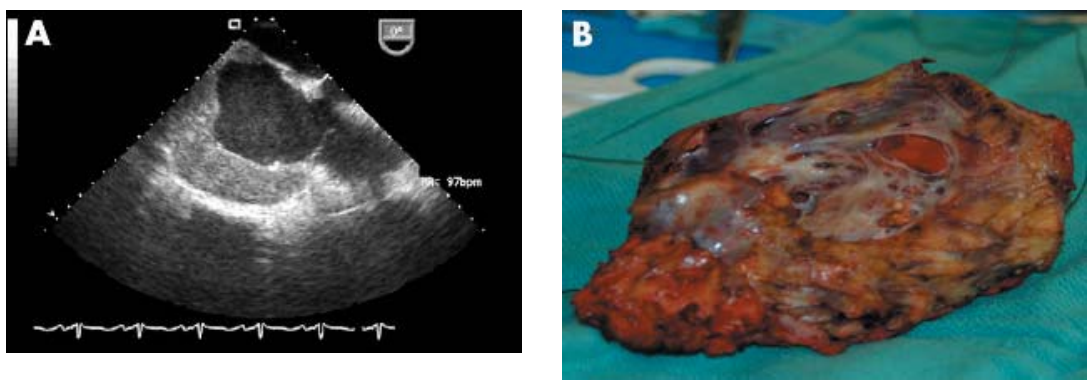

atrium with the tumour. The patient underwent an excision of the mass lesion with reconstruction of the right atrium with bovine pericardium. The gross pathology specimen of the angiosarcoma is shown in panel B. doi: $10.1136 /$ hrt.2004.048660

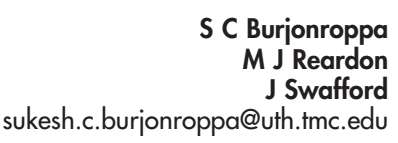

